\title{
Public Relations in health institutions
}

\author{
Eman Saleh Alkhayyal Alteneiji \\ University of Sharjah
}

Supervisor Ahmed Farouk RadwanUniversity of Sharjah

\section{Abstract}

In this study the role of public relations (PR) and Communication in health sector was highlighted. The study focuses on the role of PR in health institutions, communication strategies of PR, communication campaigns and media used by $P R$ departments in health institutions. This was studied through descriptive method using a survey that was distributed to a sample from hospitals' clients to explore there view, opinions, and evaluation about the role of $P R$ in health. Results showed that communication activities in health fields help in raising the health awareness. It also emphasized the importance of using integrated media to convey the health information and the desired health behaviors. 


\section{Introduction}

Our daily life depends on communication and public relations, as we have become in the world of unlimited communication. While there is still a kind of blurring in the role of public relations. Especially in vital sectors like health sector. As Grunig et al. (2002) stated, Public relations concept mainly entails communication, and it is abbreviated as PR. this term was first used in the United States, from where it spread to other parts of the world. According to Morgan et al. (1993), public relations is communication within the organization in an effort to achieve and realize common interests as well as a mutual understanding. The first and vital task of a public relations officer is to connect the public with the organization.

Healthcare services are administered by different regulatory authorities in the United Arab Emirates including the Ministry of Health and Prevention, Health Authority-Abu Dhabi (HAAD), the Dubai Health Authority (DHA) and the Emirates Health Authority (EHA). (www.uae-embassy.org)

\section{Literature review}

Public relations in healthcare entails communication with the health institutions with their external and internal publics. Today, public relations are used in almost every aspect of different sectors and industries, one such sector is health. Public relations in healthcare sector has been adopted in many healthcare institutions, both public and private, and this is proof enough that it plays a significant role in healthcare. Today, high cost of medical care, challenges in delivering high-quality healthcare, as well as the need of efficient management of resources and healthcare facilities emphasis the need of public relations. In the hospital, public relations are important and can play a vital role in the hospital attractiveness and reputation. The healthcare sector is changing, and this has been witnessed in the public relations department too. One of the main factors that have revolutionized public relations is technology. Many public relations officers who were used to the traditional methods of public relations are now seeing the need to adapt to the new changes in conducting their public relations activities. (Ledignham, 2006).

In the United Arab Emirates, the healthcare sector has experienced rapid growth in the last decade, and according to Draper et al. (2001), 
this is expected to continue, and this is driven by a gap between supply and demand. According to the UAE's 2021 healthcare vision, the nation plans to build a world-class healthcare system that contains effective infrastructure, expertise, and services to fulfill the growing needs of its citizens.

The healthcare sector in the UAE is quite different from that of its neighbors, in that, Abu Dhabi and Dubai won their healthcare regulators. Some of these regulators detail the Ministry of Health, Dubai Health Authority and the Health Authority of Abu Dhabi. Each entity has a different responsibility and role to play in the healthcare, these including licensing doctors, nurses and regulating the healthcare sector (AlixPartners, 2015). Although the ministry of health is a major player in the UAE healthcare sector, it is not the only provider of healthcare and this is because of the numerous initiatives that have been launched to boost the healthcare sector. For instance, the UAE recently launched a number of initiatives with the intention of promoting healthcare innovation. One of these initiatives is the Telemedicine, this entails using digital technologies to provide healthcare services from a remote location. The Abu Dhabi Telemedicine center is a joint venture between Medgate, a leading telemedicine provider based in Switzerland and Mubadala. This initiative is aimed at relieving the shortage of high-quality healthcare practitioners, and also reduces costs and gives greater access to general specialist care for patients through a number of different digital media.

According to EUAE (2020), the UAE has a comprehensive, government-funded healthcare service, comprehensive and rapidly developing, especially the private healthcare sector that delivers highquality healthcare to the population. Healthcare in the UAE is regulated at both the federal and emirate levels. Public healthcare is administered by different regulatory bodies, including the ministry of health and Prevention. The introduction of public relations in the healthcare system in the UAE provided for increased quality in healthcare services, and the public's trust increased. This created an environment for easy access to good quality healthcare across all stages, thus leading to an increase in life expectancy in the UAE up to 79 years. This level is similar to life expectancy in the United States and Europe.

34 Public relations in the UAE healthcare sector mainly focuses on the development of organizational and legal frameworks that are based 
on effective medical services to ensure that the public receives highquality medical services, thus creating a good image for both the private and public healthcare providers. Part of innovation in the UAE healthcare sector has mainly focused on the communications part under public relations. This is regarded as vital because people's perception of healthcare services also determines their trust in the healthcare providers and thus their health. This significant shift has been taking place in the last decade as the UAE opens up to the world through business and other relevant sectors.

According to a study conducted by AlixPartners (2015), showing the role of the public relations department in hospitals in the UAE, the findings revealed that public relations have become a vital part of the hospitals based in the UAE in terms of administration and development works. The results revealed that $80 \%$ of the hospitals had their own public relations departments that were directly linked to the administration. Only a small percentage of the hospitals did not see public relations as important in their organizations. As such, these organizations give the role of public relations to other departments instead.

As regards the significance of the roles and responsibilities carried out by the public relations department, this study reveals that a majority of the participants in this study underscored the great importance of supplying the administration with the required information to help in decision making. This is contrary to the findings of Akinici et al. (2004), who states that public relations officers are valued mainly because of the information they provide the top management that is vital in the overall management of the hospitals. However, the majority of participants stated that since public relations officers act as a communication link between the administration and the public, this is a vital factor. The main findings showed that the majority agree that the public relations officers play a role as expiates in diagnosing and tackling the challenges encountered by the hospital's public, they work with the administration in formulating plans and strategies, they also help in making alternative problem-solving plans, they play a vital role in aiding in the administration of the hospital, they help draw up programs and also offer help in accomplishing the same.

Moreover, $57 \%$ of participants agree that public relations officers in UAE hospitals help in establishing a strong relationship with the 
mass media, they also keep the hospital updated with the latest social, cultural and technical variables, they compose materials that are pertinent to public relations that mainly aim at improving the image of the hospitals, they also counsel the administration to abide by the structured plans and processes, and they are accountable for the success or failure of programs formulated by the public relations department in the hospital.

Looking at these findings, it is evident that public relations officers in UAE hospitals mainly conduct traditional tasks which have not developed enough to be able to cope with the new challenges and variables in the healthcare sector (AlixPartners, 2015). On top of the list of functions played by the public relations officers is the role of being the link between the administration and the internal and external public. Additionally, they also take part in decision making and formulation of management policies of the hospitals as well as receiving guests and seeing them off, and ensuring that their needs are well catered for. Nonetheless, this turns contrary to what most public relations officers consider negligible, easy and conventional roles that may thwart their potential participation in the decision-making process, formulation of organizational strategies and also formulating policies affecting the hospital.

Moreover, these findings reveal that public relations officers in UAE hospitals are yet to employ new role and perception of public relations and hence, in measuring up with the new variables and challenges. Private hospitals in Sharjah have been strongly impacted by the public relations department. Majority of private hospitals and healthcare institutions have a public relations department to fulfill the responsibilities of public relations. There is a strong interdependence between the public relations agencies and the private hospitals, which has brought about the proliferation of public relations in the international arena in UAE (Koornneef et al., 2012), as well as hospitals in Sharjah and the wider UAE.

In the last decade, the public healthcare sector in Sharjah and the wider United Arab Emirates has undergone major developments. The ministry of health had been facing major challenges in creating strong and effective relationships between the public and the hospitals. As such, 36 the public hospitals need effective two-way communication to interact with their public through research, strategic planning as well as opinion 
polls. Nonetheless, despite the rapid expansion, and proliferation that the public relations sector has enjoyed in Sharjah, there have been major structural, cultural and organizational challenges, including inadequate research and opinion polls, lack of enough qualified public relations personnel, concentration on publicity and press agentry functions, and lack of involvement of the public relations department in vital decision-making process. Nonetheless, Alix Partners (2015) conducted a research to investigate the main role played by public relations in hospitals in Sharjah, and although the sample size was small, he found out that public relations mainly engage in communication between the hospital's public and the administration and they also counsel the top management through the decision-making process. These roles are important and have played a significant role in ensuring that Sharjah residents are offered high-quality healthcare services at affordable prices over the last few years.

\section{Research problem and questions}

By reviewing previous studies, we do not find studies that clarified the role of relationships in this sector, which plays a vital and crucial role in human life and from this point we thought to study the role of relationships from a community perspective. Trying to help in developing strategies for the future of public relations in the health sector in the UAE.

This study also attempted to find answers to the following questions: 1. What is the functions of PR in government health institutions?

2. How is the public evaluate the PR and communication role and activities in health sector?

3. How is the public evaluate the PR and communication activities in both the public and private heath institutions?

4. What is the preferred media by the public to get health information and advises?

\section{Methodology}

In this research we seek to help in developing strategies for the future of public relations in the health sector in the UAE. By agreeing on the importance of public relations in our daily life and the important role public relations playing in various field especially healthcare field, this study was conducted to find out public opinions about the role of 
PR in hospitals. An online electronic questionnaire of 18 questions was developed and distributed among random sample of population in Sharjah, UAE.

\section{Sample}

Sample was selected randomly from UAE population. total sample was 101 from different age group, nationalities, gender and educational level was vary. table 1 below shows the personal information of the 101 participants.

Table 1: results / personal information of the participants

\begin{tabular}{|c|c|c|c|c|c|}
\hline \multicolumn{6}{|c|}{ Total number of valid responses is 101} \\
\hline Age groups & $18-30$ & $31-40$ & above & & total \\
\hline $\begin{array}{l}\text { number of } \\
\text { participants }\end{array}$ & 40 & 50 & 11 & & \multirow{7}{*}{101} \\
\hline Nationality & \multicolumn{2}{|c|}{ UAE } & \multicolumn{2}{|c|}{ OTHER } & \\
\hline $\begin{array}{l}\text { number of } \\
\text { participants }\end{array}$ & \multicolumn{2}{|c|}{78} & \multicolumn{2}{|c|}{23} & \\
\hline Gender & \multicolumn{2}{|c|}{ male } & \multicolumn{2}{|c|}{ female } & \\
\hline $\begin{array}{l}\text { number of } \\
\text { participants }\end{array}$ & \multicolumn{2}{|c|}{26} & \multicolumn{2}{|c|}{75} & \\
\hline educational level & $\begin{array}{c}\text { below } \\
\text { Secondary }\end{array}$ & Secondary & $\begin{array}{l}\text { Bachelor } \\
\text { degree }\end{array}$ & $\begin{array}{c}\text { Master } \\
\text { degree } \\
\text { and } \\
\text { above }\end{array}$ & \\
\hline $\begin{array}{l}\text { number of } \\
\text { participants }\end{array}$ & 5 & 14 & 59 & 23 & \\
\hline
\end{tabular}

\section{Results}

The results below clarify the perceptions of participants on the role of PR in hospitals and their opinions about health awareness campaigns done by hospitals.

\section{The importance of governmental health institutions messages:}

The results in chart no. (1) showed that $61.4 \%$ of the participants either strongly agree or agree that the awareness messages issued (38) by governmental health institutions are more important from nongovernmental health institutions, which indicates that they trust 
governmental health institutions massages and they believe that messages coming from governmental health institutions is important and should be considered. While only 13.8 they disagree on that. Chart no 1 shows the 101 response on the question that stated as "I think the awareness messages issued by governmental health institutions are more important than messages from non-governmental health institutions?"

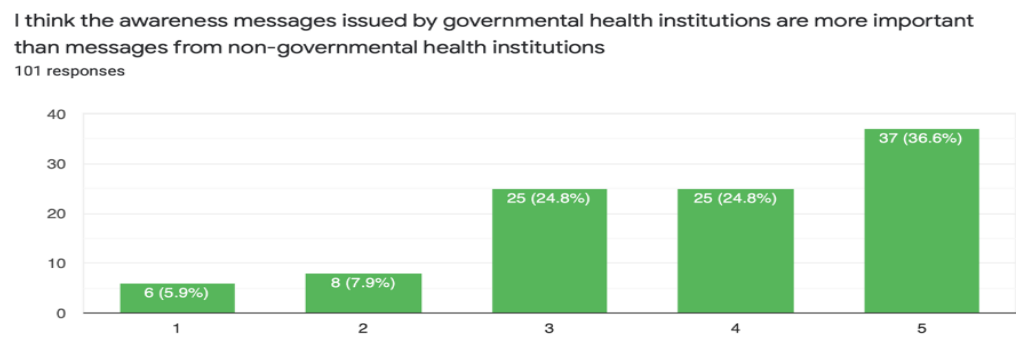

\section{Chart no 1/ the importance of governmental health message}

\section{Following the awareness messages on social media:}

The results in chart no. (2) showed that Social media does play a vital role in messages coming through it, as $67.4 \%$ of participants agreed that they are attracted to the awareness messages issued by the governmental health institutions through social media.

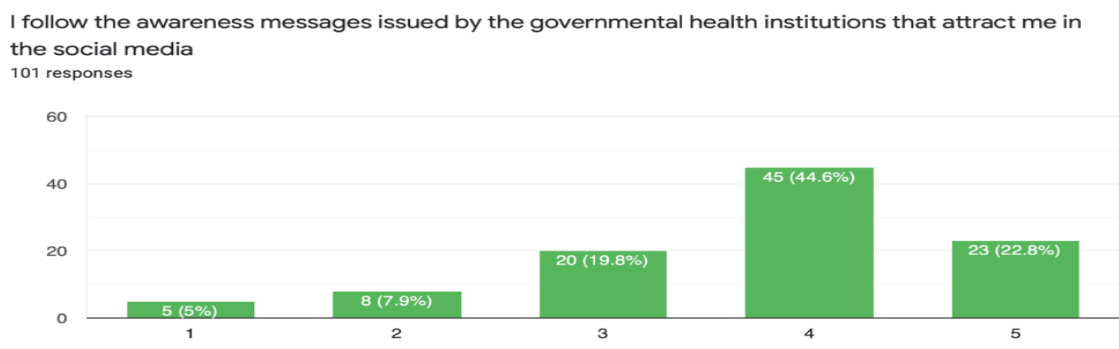

Chart no 2/ following the awareness messages on social media 


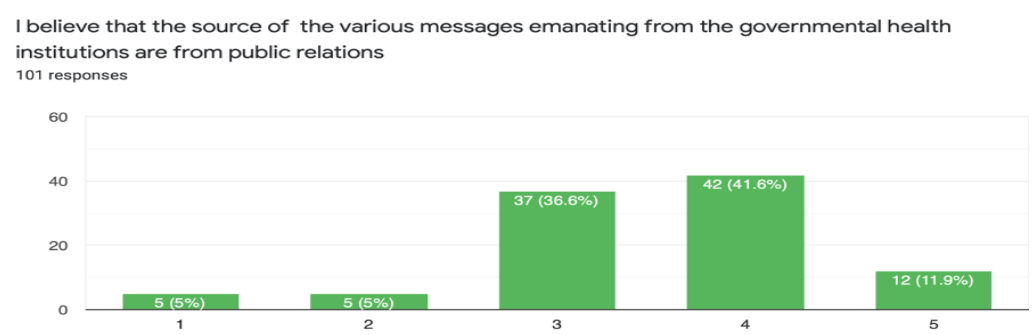

\section{Chart no 3/ public relations are source of the messages}

\section{Public relations as a source of the messages:}

The results in chart no. (3) showed that $53.5 \%$ of the participants agreed that public relations are source of the various messages from governmental health institution, however $36.6 \%$ of participants are not sure about their answer and this might be due to the lack of knowledge about the public relations role government health institutions.

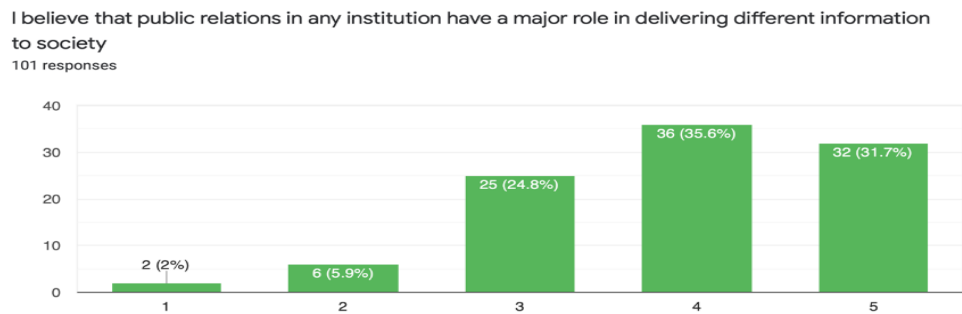

\section{Chart no 4/ public relations role of awareness}

\section{Public relations role of awareness:}

The results in chart no. (4) showed that we can clearly say that the majority of the participants did agree about the public relations role in delivering information to the society. Chart 4 above shows that $31.7 \%$ strongly agree and $35.6 \%$ agree that main role of public relations is delivering information to customers and people in society. While only $2 \%$ strongly disagree and only $5.9 \%$ disagree to that statement. $24.8 \%$ response where not sure and they don't know. PR leaders can give attention to this point, so they can do some activities to share directly

40 or indirectly with customers PR roles and responsibilities so they can provide an efficient service to everyone. 


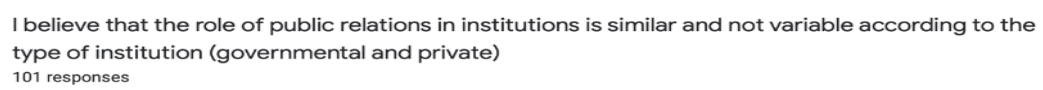

101 responses

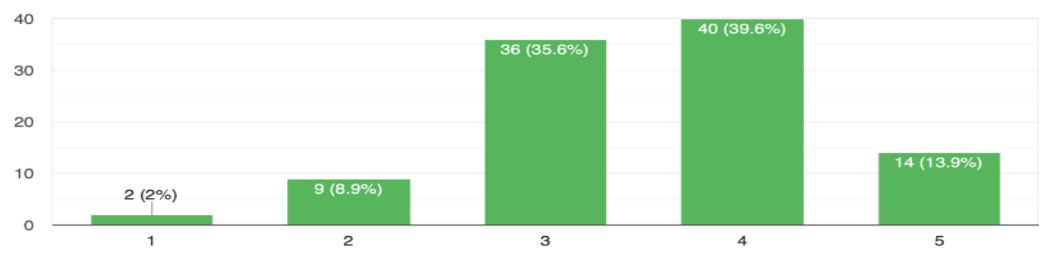

Chart no 5/ public relations role is similar every where

\section{Public relations role is similar every where:}

The results in chart no. (5) showed that Almost half of the participants agreed that the role of the public relations is similar in governmental and private sectors. Here I would like to highlight again that good number of responses where not sure and they don't know. As can be indicated from chart 5 a total of $35.6 \%$ of response they don't know.

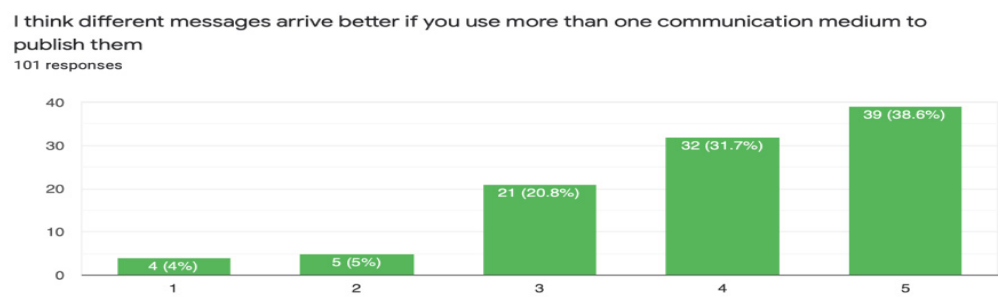

\section{Chart no 6/ using different communication medium}

\section{Using different communication medium:}

The results in chart no. (6) showed that $70.3 \%$ of the participants agreed that using different medium to deliver messages help in better arrive of messages. This indicates that it is important to have more than one source to deliver the information. And to give attention to the medium selected to deliver an important message. 


\begin{abstract}
Usually I prefer written messages more than audio or video messages, especially in crowded places such as hospitals 101 responses
\end{abstract}

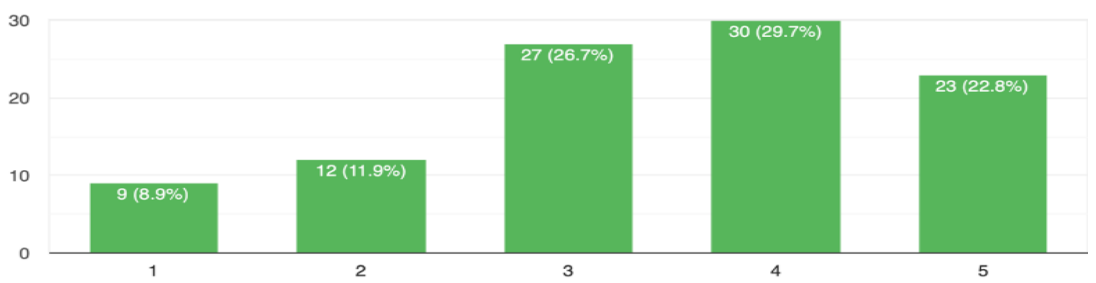

\title{
Chart no 7/ written or video massages preference
}

\section{Preference of written or video massages:}

The results in chart no. (7) showed that $26.7 \%$ of participants are not sure about this answer, however almost half of the participants agreed on the importance of having written messages in crowded places rather than audio or video messages. This might be due to the crowd noises that prevent from listening to the messages. Refer to chart 7 .

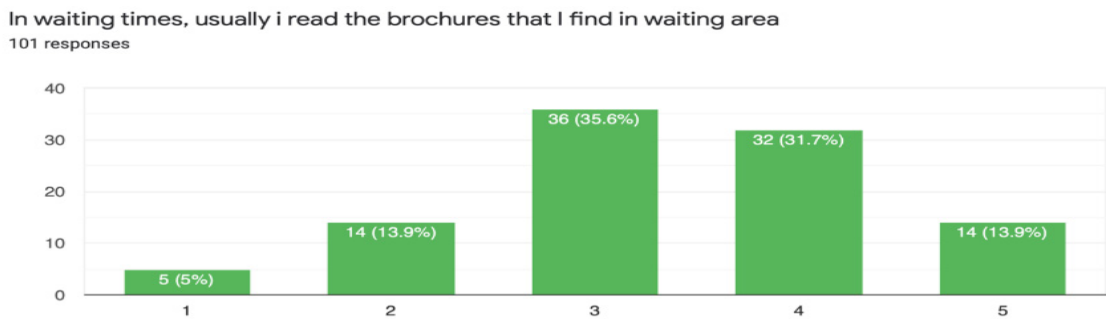

\section{Chart no 8 / reading brochures in waiting time}

\section{Reading brochures in waiting time:}

The results in chart no. (8) showed that Less than half of the participants agreed on reading the brochures in waiting areas, and this habit can be enhanced by motivating people to read in waiting areas by providing brochures to read which gives the reader valuable information. On other hands this can be used also in promoting the new services. 


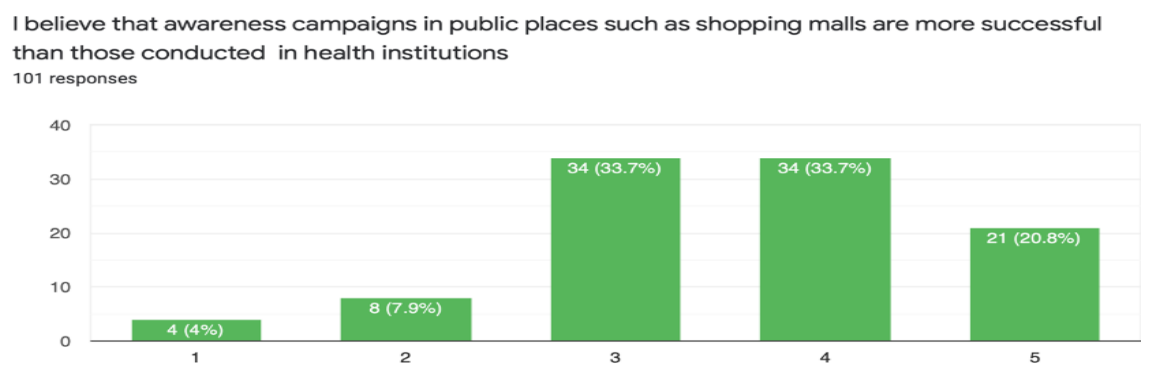

\section{Chart no 9/ awareness campaigns place}

\section{Awareness campaigns location:}

The results in chart no. (9) showed that More than half of the participants agreed that awareness campaigns in public places are more successful than health institutions, as people are more relaxed and easier to absorb information in public places such as shopping malls.

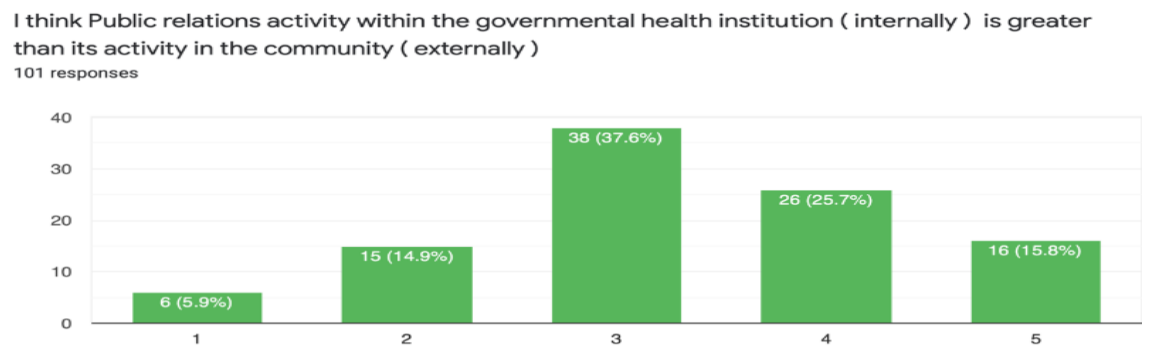

\section{Chart no 10/ comparing PR activate internally and externally}

\section{Comparing PR activate internally and externally:}

The results in chart no. (10) showed that $37.6 \%$ of the overall participants are not sure about their answer in this question, and $41.5 \%$ agreed that public relations activity within the governmental health institution is greater than its activity in the community. 


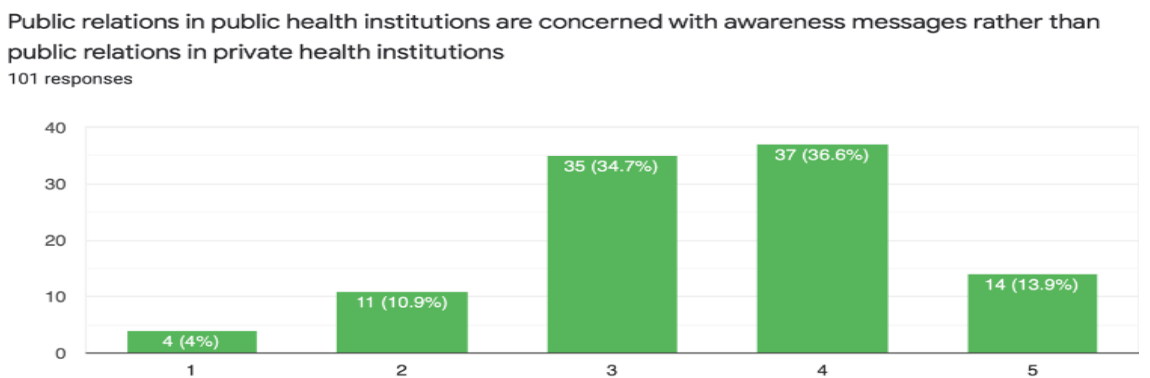

Chart no 11/ PR in public health institutions vs PR in private health institutions

PR in public health institutions vs $P R$ in private health institutions:

The results in chart no. (11) showed that Almost half of the participants agreed that public health institutions are concerned with awareness messages more than private health institutions, and reason might be that private health institutions are profitable institutions.

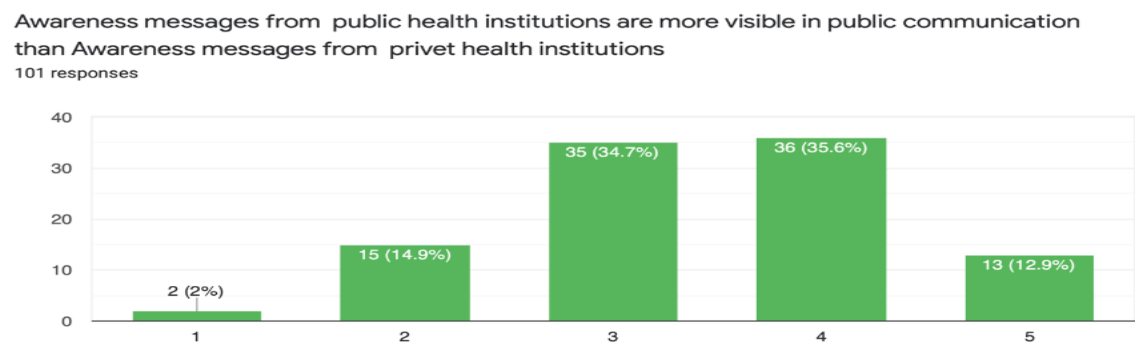

\section{Chart no 12/ visibility of awareness massages}

\section{Visibility of awareness massages:}

The results in chart no. (12) showed that $48.5 \%$ of the participants agreed that the awareness messages from public health institutions are more visible in public communication than awareness messages from private health institutions. Refer to chart 16. 
I follow health awareness campaigns

101 responses

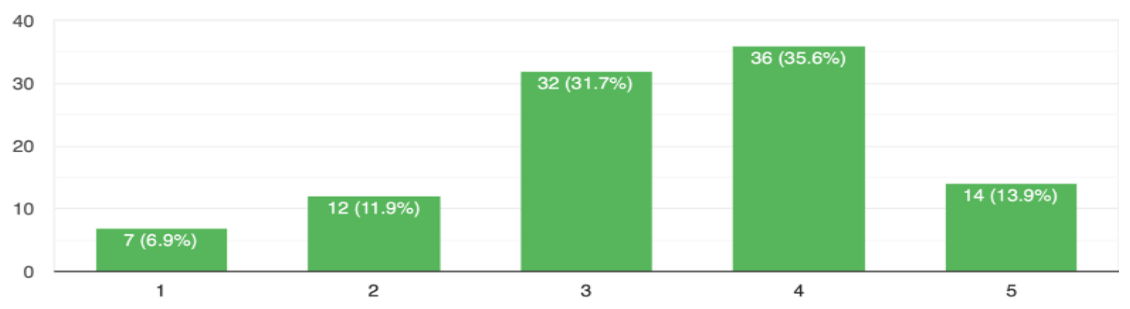

\section{Awareness campaigns influence:}

The results in chart no. (14) showed that Almost half of the participants are affected positively from the campaigns, which is again a good sign that can be enhanced. Chart 14 clearly show that only small percentage of participants do not get influenced by massages from campaigns.

\footnotetext{
I believe that awareness campaigns that rely on direct communication with the public are stronger in influencing then awareness campaigns offered by...nication such as television, radio or social media 101 responses:
}

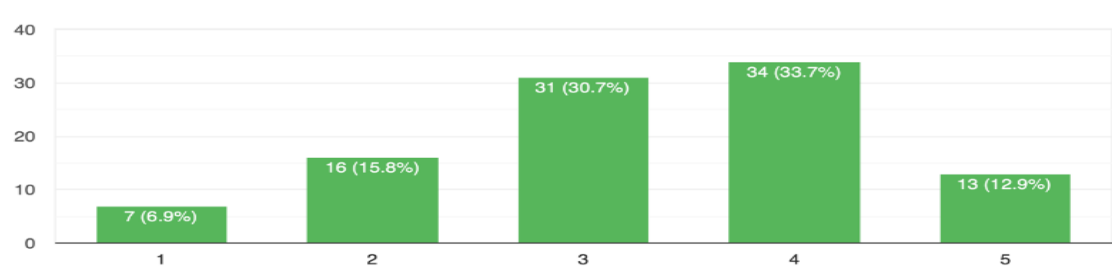

\section{Chart no 15/ awareness campaigns influence by medium}

\section{Awareness campaigns influence by medium:}

The results in chart no. (15) showed that Less than half of the participants believe that awareness campaigns that rely on direct communication with the public are stronger in influencing then awareness campaigns offered by any other means of communication such as television, radio or social media. All percentage can be figured from chart above. 


\section{I think hospital awareness campaigns reflect and promote hospital services} 101 responses

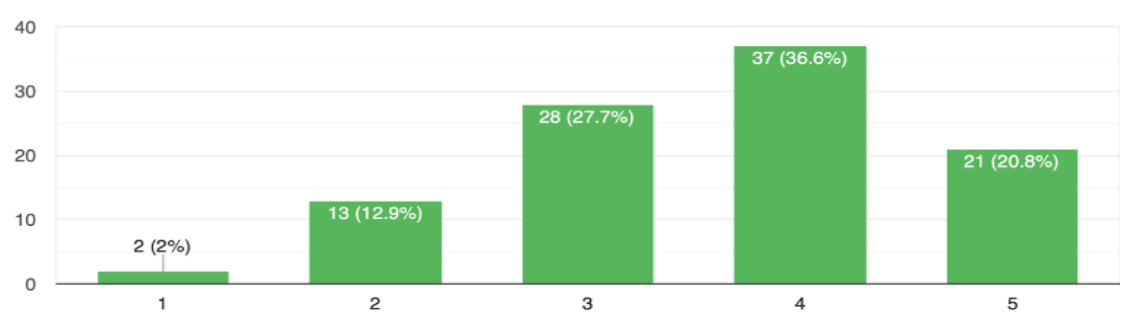

\section{Chart no 16/ promoting hospital services through campaigns}

\section{Promoting hospital services in campaigns:}

The results in chart no. (16) showed that $57.4 \%$ of participants think hospital awareness campaigns reflect and promote hospital services while just $14.9 \%$ disagree.

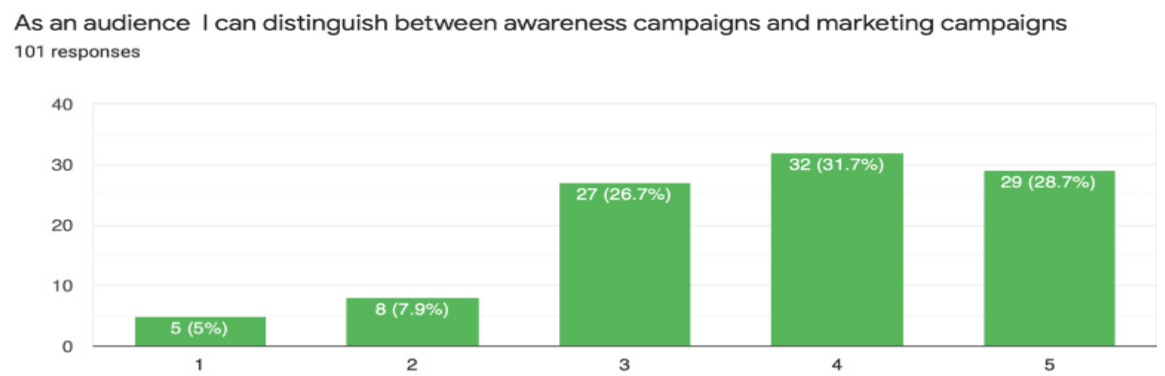

Chart no 17/ the ability to differentiate between awareness and marketing campaigns

\section{Differentiate between awareness and marketing campaigns:}

The results in chart no. (17) showed that $60.4 \%$ of the participants can distinguish between awareness campaigns and marketing campaigns, which might be because most of the participants are educated people. $5 \%$ of participants only cannot at all differentiate between awareness and marketing campaigns. 


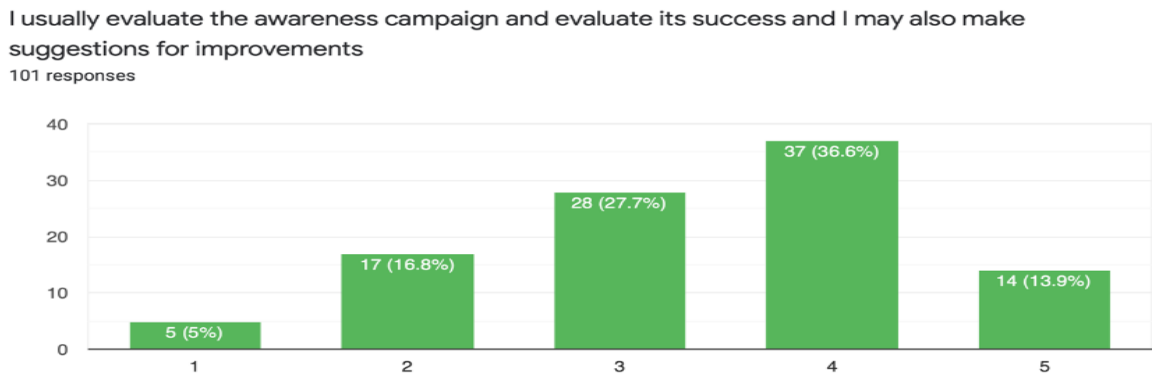

\section{Chart no 18/ participate in evaluating awareness campaigns}

\section{Evaluating awareness campaigns:}

The results in chart no. (18) showed that $50.5 \%$ of the participants agree that they can usually evaluate the awareness campaign and evaluate its success and may also make suggestions for improvements. With proper awareness campaigns and by educating people this percentage can increase. Also, I think this percentage is influenced by educational level of participants.

\section{Conclusion:}

Participants believed that public relations in any institution have a major role in delivering different information to society. The results also showed us that most of the participants prefer written messages such as brochures in the hospitals rather that other forms of voice or video messages. however, this might be because of the credibility of the written documents that usually are revised several times before printing it. Most participants agreed that awareness messages from public health institutions are more visible in public communication than awareness messages from private health institutions, and this might be due to the vast role of the government in supporting the public relation departments in the public health institutions. As public relation roles are considered costly and requires lot of effort and support from the management, which might be less in private health institutions when compared to public health institution. 


\section{Reference:}

-Akinici, F., Esatoglu, A. E., Tengilimoglu, D., and Parsons, A. (2004). Hospital choice factors: A case study in Turkey, Health Marketing Quarterly, 22 (1), 3-19.

-AlixPartners. (2015). The Healthcare Sector in the United Arab Emirates. Retrieved from: https://centres.insead.edu/ innovationpolicy/publications/documents/HealthcareBrief_ooo.pdf -Draper, M., Cohen, P., \& Buchan, H. (2001). Seeking consumer views: what use are results of hospital patient satisfaction surveys? International Journal for Quality in Health Care, 13(6), 463-468, DOI: 10.1093/intqhe/13.6.463 463-468.

-EUAE. (2020). Health Care. Retirveed from: https://www.uaeembassy.org/about-uae/health-care (Accessed March 6th 2020), (Accessed Nov16 2020)

-Grunig, L., Grunig, J., \& Dozier, D. (2002). Excellent public relations and effective organizations: A study of communication management in three countries. Mahwah, NJ: Lawrence Erlbaum.

-Koornneef, E. J., Robben, P. B., Al Seiari, M. B., \& Al Siksek, Z. (2012). Health system reform in the emirate of Abu Dhabi, United Arab Emirates. Health policy, 108(2-3), 115-121.

-Ledingham, J. A. (2006). Relationship management: A general theory of public relations. In C. H. Botan, \& V. Hazleton (Eds.), Public relations theory II (pp.465-483). Mahwah, NJ: Erlbaum.

-Morgan et al., (1993). Hospital foundations raise large amounts of money, but also raise some troublesome issues. Canadian Medical Association Journal. Toronto.

https://www.vision2021.ae/en/national-agenda-2021/list/worldclass-circle (accessed NOV 2020) 